\title{
Cosmological implications of a Relic Neutrino Asymmetry
}

\author{
Julien Lesgourgues * and Sergio Pastor ${ }^{\dagger}$ \\ SISSA-ISAS and INFN, Sezione di Trieste \\ Via Beirut 2-4,I-34013 Trieste, ITALY
}

\begin{abstract}
We consider some consequences of the presence of a cosmological lepton asymmetry in the form of neutrinos. A relic neutrino degeneracy enhances the contribution of massive neutrinos to the present energy density of the Universe, and modifies the power spectrum of radiation and matter. Comparing with current observations of cosmic microwave background anisotropies and large scale structure, we derive some constraints on the neutrino degeneracy and on the spectral index in the case of a flat Universe with a cosmological constant.
\end{abstract}

\section{INTRODUCTION}

It is generally assumed that our Universe contains an approximately equal amount of leptons and antileptons. The lepton asymmetry would be of the same order as the baryon asymmetry, which is very small as required by Big Bang Nucleosynthesis (BBN) considerations. The existence of a large lepton asymmetry is restricted to be in the form of neutrinos from the requirement of universal electric neutrality, and the possibility of a large neutrino asymmetry is still open. From a particle physics point of view, a lepton asymmetry can be generated by an Affleck-Dine mechanism [1] without producing a large baryon asymmetry (see ref. [2] for a recent model), or even by active-sterile neutrino oscillations after the electroweak phase transition (but in this last case, it might not be of order unity) [3]. This lepton asymmetry can postpone symmetry restoration in non-supersymmetric or supersymmetric models [4] (note that this is also true for other charges [5]).

In this paper we study some cosmological implications of relic degenerate neutrinost]. We do not consider any specific model for generating such an asymmetry, and just assume that it was created well before neutrinos decouple from the rest of the plasma. An asymmetry of order one or larger can have crucial effects on the global evolution of the Universe. Among other effects, it changes the decoupling temperature of neutrinos, the primordial production of light elements at BBN, the time of equality between radiation and matter, or the contribution of relic neutrinos to the present energy density of the Universe. The latter changes affect the evolution of perturbations in the Universe. We focus on the anisotropies of the Cosmic Microwave Background (CMB), and on the distribution of Large Scale Structure (LSS). We calculate the power spectrum of both quantities, in the case of massless degenerate neutrinos, and also for neutrinos with a mass of $0.07 \mathrm{eV}$, as suggested to explain the experimental evidence of atmospheric neutrino oscillations at Super-Kamiokande [6]. The cosmological implications of neutrinos with such a small mass are known to be very small, but we will see that this conclusion is modified if a large neutrino degeneracy exists. We also include in our analysis the possibility that the dominant contribution to the present energy density in the Universe is due to a cosmological constant: $\Omega_{\Lambda} \sim 0.6-0.7$, keeping the Universe flat $\left(\Omega_{0}+\Omega_{\Lambda}=1\right)$, as suggested by recent observations (see [7] and references therein).

The effect of neutrino degeneracy on the LSS power spectrum was studied in ref. [8], as a way of improving the agreement with observations of mixed dark matter models with eV neutrinos, in the case of high values of the Hubble parameter. Also, Adams \& Sarkar [9] calculated the CMB anisotropies and the matter power spectrum, and compared them with observations in the $\Omega_{\Lambda}=0$ case for massless degenerate neutrinos. More recently, Kinney \& Riotto [10] also calculated the CMB anisotropies for massless degenerate neutrinos in the $\Omega_{\Lambda}=0.7$ case.

This paper is organized as follows. In section [1], we calculate the contribution of massive degenerate neutrinos to the present energy density of the Universe. In section III, we explain how to calculate the power spectra, with the help of the code cmbfast 11. In section IV], we discuss the effect of the degeneracy on CMB anisotropies and the matter power spectrum, both for massless neutrinos and $m_{\nu}=0.07 \mathrm{eV}$. Finally, in section $\mathrm{V}$, we derive some

\footnotetext{
*E-mail: lesgour@sissa.it

${ }^{\dagger}$ E-mail: pastor@sissa.it

${ }^{1}$ Here, by degeneracy, we mean that there exists a large neutrino-antineutrino asymmetry, or vice versa, and not a degeneracy in the mass sense.
} 
constraints on the neutrino degeneracy from CMB and LSS data, in the particular case of a flat Universe with an arbitrary cosmological constant and for standard values of other cosmological parameters.

\section{ENERGY DENSITY OF MASSIVE DEGENERATE NEUTRINOS}

The energy density of one species of massive degenerate neutrinos and antineutrinos, described by the distribution functions $f_{\nu}$ and $f_{\bar{\nu}}$, is (here and throughout the paper we use $\hbar=c=k_{B}=1$ units)

$$
\rho_{\nu}+\rho_{\bar{\nu}}=\int \frac{d^{3} \vec{p}}{(2 \pi)^{3}} E_{\nu}\left(f_{\nu}(p)+f_{\bar{\nu}}(p)\right)=\frac{1}{2 \pi^{2}} \int_{0}^{\infty} d p p^{2} \sqrt{p^{2}+m_{\nu}^{2}}\left(f_{\nu}(p)+f_{\bar{\nu}}(p)\right),
$$

valid at any moment. Here $p$ is the magnitude of the 3 -momentum and $m_{\nu}$ is the neutrino mass.

When the early Universe was hot enough, the neutrinos were in equilibrium with the rest of the plasma via the weak interactions. In that case the distribution functions $f_{\nu}$ and $f_{\bar{\nu}}$ changed with the Universe expansion, keeping the form of a Fermi-Dirac distribution,

$$
f_{\nu}(p)=\frac{1}{\exp \left(\frac{p}{T_{\nu}}-\frac{\mu}{T_{\nu}}\right)+1} \quad f_{\bar{\nu}}(p)=\frac{1}{\exp \left(\frac{p}{T_{\nu}}+\frac{\mu}{T_{\nu}}\right)+1}
$$

Here $\mu$ is the neutrino chemical potential, which is nonzero if a neutrino-antineutrino asymmetry has been previously produced. Later the neutrinos decoupled when they were still relativistic?, and from that moment the neutrino momenta just changed according to the cosmological redshift. If $a$ is the expansion factor of the Universe, the neutrino momentum decreases keeping ap constant. At the same time the neutrino degeneracy parameter $\xi \equiv \mu / T_{\nu}$ is conserved, with a value equal to that at the moment of decoupling. Therefore one can still calculate the energy density of neutrinos now from eq. (11) and eq. (2), replacing $\mu / T_{\nu}$ by $\xi$ and $p / T_{\nu}$ by $p /\left(y_{\nu} T_{0}\right)$, where $T_{0} \simeq 2.726 \mathrm{~K}$ and $y_{\nu}$ is the present ratio of neutrino and photon temperatures, which is not unity because once decoupled the neutrinos did not share the entropy transfer to photons from the successive particle annihilations that occurred in the early Universe. In the standard case, the massless non-degenerate neutrinos decoupled just before the electron-positron pairs annihilated to photons, from which one gets the standard factor $y_{\nu}=(4 / 11)^{1 / 3}$.

In the presence of a significant neutrino degeneracy $\xi$ the decoupling temperature $T(\xi)$ is higher than in the standard case 12, 13. The reaction rate $\Gamma$ of the weak processes, that keep the neutrinos in equilibrium with the other species, is reduced because some of the initial or final neutrino states will be occupied. The authors of ref. [13] used the Boltzmann equation to calculate $\Gamma$ for the process $\nu_{d}+\bar{\nu}_{d} \leftrightarrow e^{+}+e^{-}$(here $\nu_{d}$ denotes degenerate neutrinos), including the corresponding Fermi blocking factors. It was found that the neutrino decoupling temperature is $T_{d e c}(\xi) \approx$ $0.2 \xi^{2 / 3} \exp (\xi / 3) \mathrm{MeV}$ (for $\nu_{\mu}$ or $\nu_{\tau}$ ). Therefore if $\xi$ is large enough, the degenerate neutrinos decouple before the temperature of the Universe drops below the different mass thresholds, and are not heated by the particle-antiparticle annihilations. The ratio of neutrino and photon temperatures is thus reduced accordingly.

The present contribution of these degenerate neutrinos to the energy density of the Universe can be parametrized as $\rho_{\nu}=10^{4} h^{2} \Omega_{\nu} \mathrm{eV} \mathrm{cm}{ }^{-3}$, where $\Omega_{\nu}$ is the neutrino energy density in units of the critical density $\rho_{c}=3 H^{2} M_{P}^{2} / 8 \pi$, $M_{P}=1.22 \times 10^{19} \mathrm{GeV}$ is the Planck mass and $H=100 h \mathrm{Km} \mathrm{s}^{-1} \mathrm{Mpc}^{-1}$ is the Hubble parameter. The value of $\rho_{\nu}$ can be calculated as a function of the neutrino mass and the neutrino degeneracy $\xi$, or equivalently the present neutrino asymmetry $L_{\nu}$ defined as the following ratio of number densities

$$
L_{\nu} \equiv \frac{n_{\nu}-n_{\bar{\nu}}}{n_{\gamma}}=\frac{1}{12 \zeta(3)} y_{\nu}^{3}\left[\xi^{3}+\pi^{2} \xi\right]
$$

We show in figures 1 and 2 the contours in the $\left(m_{\nu}, L_{\nu}\right)$ and $\left(m_{\nu}, \xi\right)$ planes that correspond to some particular values of $h^{2} \Omega_{\nu}$. One can see from the figures that there are two limits: massive non-degenerate neutrinos and massless degenerate neutrinos. The first case corresponds to the vertical lines, when one recovers the well-known bound on the neutrino mass $m_{\nu} \lesssim 46 \mathrm{eV}$ for $h^{2} \Omega_{\nu}=0.5$. On the other hand, for very light neutrinos, the horizontal lines set a maximum value on the neutrino degeneracy, that would correspond to a present neutrino chemical potential

\footnotetext{
${ }^{2}$ Unless the neutrino mass is comparable to the decoupling temperature, $\mathcal{O}\left(m_{\nu}\right) \sim \mathrm{MeV}$.

${ }^{3}$ Here we assume $\xi>0$, but the results are also valid for $\xi<0$ provided that $\xi$ and $L_{\nu}$ are understood as moduli.
} 
$\mu_{0} \lesssim 7.4 \times 10^{-3} \mathrm{eV}$, also for $h^{2} \Omega_{\nu}=0.5$. In the intermediate region of the figures the neutrino energy density is $\rho_{\nu} \simeq m_{\nu} n_{\nu}(\xi)$ and the contours follow roughly the relations

$$
\begin{gathered}
L_{\nu}\left(\frac{m_{\nu}}{\mathrm{eV}}\right) \simeq 24.2 h^{2} \Omega_{\nu} \\
\left(\pi^{2} \xi+\xi^{3}\right)\left(\frac{m_{\nu}}{\mathrm{eV}}\right) \simeq \frac{350}{y_{\nu}^{3}} h^{2} \Omega_{\nu}
\end{gathered}
$$

A similar calculation has been recently performed in reference 14. However the difference between neutrino and photon temperatures was not properly taken into account for large $\xi$. It was argued that, since the number density of highly degenerate neutrinos is larger than in the non-degenerate case, the neutrinos would have been longer in thermal contact with $e^{+} e^{-}$, therefore sharing with photons the entropy release. However this is not the case [13] as we discussed before.

The presence of a neutrino degeneracy can modify the outcome of Big Bang Nucleosynthesis (for a review see [15]). First a larger neutrino energy density increases the expansion rate of the Universe, thus enhancing the primordial abundance of ${ }^{4} \mathrm{He}$. This is valid for a nonzero $\xi$ of any neutrino flavor. In addition if the degenerate neutrinos are of electron type, they have a direct influence over the weak processes that interconvert neutrons and protons. This last effect depends on the sign of $\xi_{\nu_{e}}$. Both effects may be simultaneously important and it could be possible in principle to explain the observed primordial abundances with a large baryon density, $\Omega_{B} h^{2} \approx 1$ [12,13]. However this possibility is ruled out by the fact that in that case our Universe would have been radiation dominated during a longer period and the observed large-scale structure would be difficult to explain. From BBN one gets the following constraint [13]

$$
-0.06 \lesssim \xi_{\nu_{e}} \lesssim 1.1
$$

while a sufficiently long matter dominated epoch requires

$$
\left|\xi_{\nu_{\mu}, \nu_{\tau}}\right| \lesssim 6.9
$$

This estimate from [13] agrees with our analysis in section $\square$. Assuming that the degenerate neutrinos are $\nu_{\mu}$ or $\nu_{\tau}$, this places a limit on the degeneracy as shown by the horizontal line in figures 1 and 2 .

\section{POWER SPECTRA CALCULATION}

We compute the power spectra of CMB anisotropies and large-scale structure using the Boltzmann code cmbfast by Seljak \& Zaldarriaga [11], adapted to the case of one family of degenerate neutrinos $(\nu, \bar{\nu})$, with mass $m_{\nu}$ and degeneracy parameter $\xi$. Let us first review the required modifications. We use the notations of Ma \& Bertschinger [16], and for all issues not specific to our case, we refer the reader to this review.

Background quantities can be rewritten in terms of two dimensionless parameters $(M, Q)$

$$
M=\frac{m_{\nu}}{T_{\nu 0}}=\frac{m_{\nu}(\mathrm{eV})}{8.6170 \times 10^{-5} \times(4 / 11)^{1 / 3} T_{0}(\mathrm{~K})}, \quad Q=\frac{a p}{T_{\nu 0}}
$$

(we are assuming $y_{\nu}=(4 / 11)^{1 / 3}$, and therefore $\xi \leq 12$ [13]; the scale factor is defined so that $a=1$ today). For SuperKamiokande neutrinos with $m_{\nu}=0.07 \mathrm{eV}, M \simeq 417$. We then get for the mean density, pressure and phase-space distributions

$$
\begin{aligned}
\bar{\rho}_{\nu}+\bar{\rho}_{\bar{\nu}} & =\frac{T_{\nu}^{4}}{2 \pi^{2}} \int Q^{2} d Q \sqrt{Q^{2}+a^{2} M^{2}}\left(f_{\nu}(Q)+f_{\bar{\nu}}(Q)\right), \\
\bar{P}_{\nu}+\bar{P}_{\bar{\nu}} & =\frac{T_{\nu}^{4}}{6 \pi^{2}} \int Q^{2} d Q \frac{Q^{2}}{\sqrt{Q^{2}+a^{2} M^{2}}}\left(f_{\nu}(Q)+f_{\bar{\nu}}(Q)\right), \\
f_{\nu}(Q) & =\frac{1}{e^{Q-\xi}+1}, \quad f_{\bar{\nu}}(Q)=\frac{1}{e^{Q+\xi}+1} .
\end{aligned}
$$

In the case of massive degenerate neutrinos, these integrals must be calculated for each value of the scale factor, and also at the beginning of the code in order to find $\Omega_{\nu}$ today. On the other hand, for massless neutrinos, there is an exact analytic solution 


$$
\bar{\rho}_{\nu}+\bar{\rho}_{\bar{\nu}}=3\left(\bar{P}_{\nu}+\bar{P}_{\bar{\nu}}\right)=\frac{7}{8} \frac{\pi^{2}}{15} T_{\nu}^{4}\left[1+\frac{30}{7}\left(\frac{\xi}{\pi}\right)^{2}+\frac{15}{7}\left(\frac{\xi}{\pi}\right)^{4}\right] .
$$

So, if we define an effective number of massless neutrino families $N_{\text {eff }} \equiv 3+30 / 7(\xi / \pi)^{2}+15 / 7(\xi / \pi)^{4}$, the mean density and pressure for all neutrinos will be given by these ones for one massless non-degenerate family, multiplied by $N_{\text {eff }}$.

Let us now consider perturbed quantities. We define $\Psi_{\nu}$ and $\Psi_{\bar{\nu}}$, the perturbations of the phase space distribution for $\nu$ and $\bar{\nu}$, through

$$
\begin{aligned}
\delta f_{\nu}(\vec{x}, Q, \hat{n}, \tau) & =f_{\nu}(Q) \Psi_{\nu}(\vec{x}, Q, \hat{n}, \tau) \\
\delta f_{\bar{\nu}}(\vec{x}, Q, \hat{n}, \tau) & =f_{\bar{\nu}}(Q) \Psi_{\bar{\nu}}(\vec{x}, Q, \hat{n}, \tau)
\end{aligned}
$$

( $\hat{n}$ is the momentum direction: $\vec{p} \equiv p \hat{n}$ ). For our purpose, which is to integrate the linearized Einstein equations, it can be shown that only the following linear combination is relevant

$$
\Psi \equiv \frac{f_{\nu} \Psi_{\nu}+f_{\bar{\nu}} \Psi_{\bar{\nu}}}{f_{\nu}+f_{\bar{\nu}}}
$$

Using the Boltzmann equations for $\Psi_{\nu}$ and $\Psi_{\bar{\nu}}$, it is straightforward to show that the evolution of $\Psi$ (in Fourier space and in the synchronous gauge, see [16], eq. (40)) obeys

$$
\frac{\partial \Psi}{\partial \tau}+i \frac{Q}{\sqrt{Q^{2}+a^{2} M^{2}}}(\vec{k} . \hat{n}) \Psi+\frac{d \ln \left(f_{\nu}+f_{\bar{\nu}}\right)}{d \ln Q}\left[\dot{\eta}-\frac{\dot{h}+6 \dot{\eta}}{2}(\hat{k} . \hat{n})^{2}\right]=0 .
$$

This equation depends on $\xi$ only through the last term, which is the gravitational source term.

In the case $\xi=0$, the quantity $\left(d \ln \left(f_{\nu}\right) / d \ln Q\right)$ has a simple interpretation: it is the $Q$-dependence of a planckian perturbation of the phase space distribution. In other words, a shift of the blackbody temperature $\Delta T / T(\vec{x}, \hat{n}, \tau)$ corresponds to a perturbation

$$
\Psi(\vec{x}, Q, \hat{n}, \tau)=-\frac{\Delta T}{T}(\vec{x}, \hat{n}, \tau) \frac{d \ln \left(f_{\nu}\right)}{d \ln Q} .
$$

Since the gravitational source term in the Boltzmann equation is proportional to this quantity, the planckian shape is unaltered for massless neutrinos, and also for massive neutrinos when they are still relativistic (indeed, when $Q^{2} \gg a^{2} M^{2}$, the $Q$-dependence of the Boltzmann equation (12) vanishes in the second term, and remains only in the third term). When $\xi \neq 0$, the source term in eq. (12) is proportional to

$$
\frac{d \ln \left(f_{\nu}+f_{\bar{\nu}}\right)}{d \ln Q}=-\frac{Q(1+\operatorname{ch} \xi \operatorname{ch} Q)}{\left(\operatorname{ch} \xi+\mathrm{e}^{-Q}\right)(\operatorname{ch} \xi+\operatorname{ch} Q)} .
$$

When neutrinos are still relativistic, $\Psi$ is proportional to this quantity, even if it cannot be simply interpreted in terms of blackbody temperature perturbations.

We can now specify all the changes required in cmbfast, first in the case of massive degenerate neutrinos. As usual, $\Psi$ can be expanded in a Legendre series: $\Psi=\sum_{l=0}^{\infty}(-i)^{l}(2 l+1) \Psi_{l} P_{l}$. It is easy to show that for each multipole $\Psi_{l}$, the evolution equation and the initial condition are both identical to those of the non-degenerate case, provided that we replace $\left(d \ln \left(f_{\nu}\right) / d \ln Q\right)$ by eq. (14). So, in summary, one only needs to modify the homogeneous phasespace distribution, its logarithmic derivative with respect to $Q$, and the initial calculation of $\Omega_{\nu}$. Also, in order to obtain a good precision in the CMB anisotropy spectra, one must set $l=5$ for the number of multipoles $\Psi_{l}$ to be time-integrated. For transfer functions, the value $l=25$ proposed by the code is sufficient.

In the case of massless degenerate neutrinos, the situation is even simpler. The $Q$-dependence of the Boltzmann equation can be integrated away, just like in the non-degenerate case. For this purpose, we must introduce the $Q$-independent variable $F_{\nu}$

$$
F_{\nu}(\vec{k}, \hat{n}, \tau) \equiv \frac{\int Q^{3} d Q\left(f_{\nu}+f_{\bar{\nu}}\right) \Psi}{\int Q^{3} d Q\left(f_{\nu}+f_{\bar{\nu}}\right)} \equiv \sum_{l=0}^{\infty}(-i)^{l}(2 l+1) F_{\nu l} P_{l},
$$

and integrate by part the last term in equation (12). The multipoles $F_{\nu l}$ are exactly identical for degenerate and non-degenerate massless neutrinos, because they share the same evolution equations and initial conditions. So, the effect of $\xi$ arises only through the background quantities in eq. (8) and is completely described by introducing an effective number of massless neutrinos. 


\section{RESULTS}

First, as a consistency check, we compute CMB anisotropies and transfer functions for different values of $\xi$, choosing a very small mass $m_{\nu} \leq 0.001 \mathrm{eV}$. We check that the results match exactly those obtained with the unmodified version of cmbfast, when the appropriate effective neutrino number $N_{\text {eff }}$ is specified.

The effect of $\xi$ and $m_{\nu}$ on the CMB anisotropy spectrum can be seen in figure 3. We choose a set of cosmological parameters $\left(h=0.65, \Omega_{b}=0.05, \Omega_{\Lambda}=0.70, \Omega_{C D M}=1-\Omega_{b}-\Omega_{\nu}-\Omega_{\Lambda}, Q_{r m s-p s}=18 \mu \mathrm{K}\right.$, flat primordial spectrum, no reionization, no tensor contribution), and we vary $\xi$ from 0 to 5 , both in the case of massless degenerate neutrinos (solid lines) and degenerate neutrinos with $m_{\nu}=0.07 \mathrm{eV}$ (dashed lines). Let us first comment the massless case. The main effect of $\xi$ is to boost the amplitude of the first peakf. Indeed, increasing the energy density of radiation delays matter-radiation equality, which is known to boost the acoustic peaks 17 (the same explanation holds for the effect of $\Omega_{\Lambda}$ in flat models). For the same reason, all peaks are shifted to higher multipoles, by a factor $\left(\left(1+a_{e q} / a_{*}\right)^{1 / 2}-\left(a_{e q} / a_{*}\right)^{1 / 2}\right)^{-1}$ [17] $\left(a_{e q}\right.$ is the scale factor at equality, and increases with $\xi$, while the recombination scale factor $a_{*}$ is almost independent of the radiation energy density). Secondary peaks are then more affected by diffusion damping at large $l$, and their amplitude can decrease with $\xi$.

In the case of degenerate neutrinos with $m_{\nu}=0.07 \mathrm{eV}$, the results are quite similar in first approximation. Indeed, the effects described previously depend on the energy density of neutrinos at equality. At that time, they are still relativistic, and identical to massless neutrinos with equal degeneracy parameter. However, with a large degeneracy, $\Omega_{\nu}$ today becomes significant: for $\xi=5$, one has $\Omega_{\nu}=0.028$, i.e. the same order of magnitude as $\Omega_{b}$. Since we are studying flat models, $\Omega_{\nu}=0.028$ must be compensated by less baryons, cold dark matter $(\mathrm{CDM})$ or $\Omega_{\Lambda}$. In our example, $\Omega_{b}$ and $\Omega_{\Lambda}$ are fixed, while $\Omega_{C D M}$ slightly decreases. This explains the small enhancement of the first peak compared to the massless case. Even if this effect is indirect, it is nevertheless detectable in principle (even if one does not impose the flatness condition, the effect of $\Omega_{\nu}$ will be visible through a modification of the curvature). In figure 3 , for $\xi=0$, the first peak maximum is enhanced by only $0.37 \%$, while for $\xi=5$, there is an increase of $3.4 \%$, detectable by the future satellite missions $M A P$ and Planck, unless there are large parameter degeneracies. It is well-known that such degeneracies are generally removed when CMB and LSS data are combined for parameter extraction [18].

We plot in figure 4 the power spectrum $P(k)$ for the same models as in figure 3 , normalized on large scales to COBE. The effect of both parameters $\xi$ and $m_{\nu}$ is now to suppress the power on small scales. Indeed, increasing $\xi$ postpones matter-radiation equality, allowing less growth for fluctuations crossing the Hubble radius during radiation domination. Adding a small mass affects the recent evolution of fluctuations, and has now a direct effect: when the degenerate neutrinos become non-relativistic, their free-streaming suppresses growth of fluctuations for scales within the Hubble radius. For non-degenerate neutrinos, this effect is known to reduce power on those scales by a relative amount $\Delta P / P \sim 8 \Omega_{\nu} / \Omega_{0}$ [19] (we introduced $\Omega_{0}=1-\Omega_{\Lambda}$ ). So, even with $m_{\nu}=0.07 \mathrm{eV}$ and $\xi=0$, it is significant, especially at low $\Omega_{0}$. In the models of figure $4, P(k)$ decreases by $\sim 5 \%$, in agreement with the theoretical prediction $\left(\Omega_{\nu}=1.8 \times 10^{-3}, \Omega_{0}=0.3\right)$. However, at $\xi=5$ (i.e. $\left.\Omega_{\nu}=0.028\right)$, this effect is even larger: $P(k)$ decreases by a factor 2.2 , instead of an expected 1.7. This effect is likely related to the phase-space distribution of neutrinos with a chemical potential: their average momentum is shifted to larger values, making the free-streaming suppression mechanism even more efficient.

Let us compare our results with those of previous works. The effect of $\xi$ on the CMB for massless neutrinos and $\Omega_{\Lambda}=0$ is the same as that one found in [9]. We also agree with the revised results in [10].

\section{COMPARISON WITH OBSERVATIONS}

Since the degeneracy increases dramatically the amplitude of the first CMB peak, we expect large $\xi$ values to be disfavored in the case of cosmological models known to predict a fairly high peak. On the other hand, a high $\xi$ is likely to be allowed (or even favored) for models that predict systematically a low peak, unless a large scalar spectral index $n \geq 1.2$ (blue tilt) is invoked. For instance, the degeneracy is likely to be favored by: (i) a large contribution of tensor perturbations; (ii) a significant effect from reionization; (iii) a low baryon density; (iv) a large $h(h \geq 0.7$ ); (v) flat models with $\Omega_{\Lambda} \leq 0.6$; etc. For such models, the peak amplitude can be boosted by $\xi$, keeping $n$ close to one, which is more natural from the point of view of inflation. However, a careful case-by-case analysis is required,

\footnotetext{
${ }^{4}$ In fact, this is not true for very large values of $\xi$. In such cases, recombination can take place still at the end of radiation domination, and anisotropies are suppressed. For our choice of cosmological parameters, this happens for $\xi \gtrsim 7$, but in such a case the location of the first peak is $l \gtrsim 450$, and the matter power spectrum is strongly suppressed.
} 
since the effects of $\xi$ and $n$ on CMB and LSS spectra are far from being equivalent. Our goal here is not to explore systematically all possibilities, but to briefly illustrate how $\xi$ can be constrained by current observations for flat models with different values of $\Omega_{\Lambda}$. Recent results from supernovae [20], combined with CMB constraints, favor flat models with $\Omega_{\Lambda} \sim 0.6-0.7$ []].

We choose a flat model with $h=0.65, \Omega_{b}=0.05, Q_{r m s-p s}=18 \mu \mathrm{K}$, no reionization and no tensor contribution, and look for the allowed region in the space of free parameters $\left(\Omega_{\Lambda}, \xi, n\right)$. The allowed region will not be defined using a maximum likelihood analysis, but with the more conservative technique called "concordance" by Wang et al. [21], which consists in taking the intersection of regions allowed by each experiment.

For simplicity, we take into account only a few constraints on the matter power spectrum, known to be representative of the large amount of available data: the value of $\sigma_{8}$ (the variance of mass fluctuations in a sphere of radius $R=8 h^{-1} \mathrm{Mpc}$ ) given for flat models in [22], at $95 \%$ confidence level (CL) APM redshift survey [24], at scales well within the linear regime, also with $95 \%$ CLf; and finally, the constraint on bulk velocity at $R=50 \mathrm{~h}^{-1} \mathrm{Mpc}$ [25], taking into account the cosmic variance. Except for the updated $\sigma_{8}$ constraint, we use exactly the same experimental tests as in [26, and refer the reader to this paper for details. For CMB data, we perform a $\chi^{2}$ analysis based on 19 experimental points and window functions, taking into account the Saskatoon calibration uncertainty, in the way suggested by [27]. The list of data that we use is given in [26], and again allowed regions correspond to $95 \% \mathrm{CL} 7$. We do not take into account the most recent experiments, for which window functions are still unpublished; they are anyway in good agreement with the data considered here.

We plot in figure 0 the LSS and CMB allowed regions in $(\xi, n)$ parameter space, corresponding to $\Omega_{\Lambda}=0,0.5,0.6,0.7$. For $\Omega_{\Lambda}=0.5-0.7$, the LSS window just comes out of $\sigma_{8}$ limits. For $\Omega_{\Lambda}=0$, the lower LSS constraint is from $\sigma_{8}$, and the upper one from APM data. In the case of degenerate neutrinos with $m_{\nu}=0.07 \mathrm{eV}$, the windows are slightly shifted at large $\xi$, since, as we saw, the effect of $\xi$ is enhanced (dotted lines on the figure). The CMB allowed regions do not show this distinction, given the smallness of the effect and the imprecision of the data. One can immediately see that LSS and CMB constraints on $n$ are shifted in opposite direction with $\xi$ : indeed, the effects of $\xi$ and $n$ both produce a higher CMB peak, while to a certain extent they compensate each other in $P(k)$. So, for $\Omega_{\Lambda}=0.7$, a case in which a power spectrum normalized to both $\mathrm{COBE}$ and $\sigma_{8}$ yields a too high peak 8 , a neutrino degeneracy can only make things worst. On the other hand, for $\Omega_{\Lambda}=0.5-0.6$, a good agreement is found up to $\xi \simeq 3$.

Let us finally consider the $\Omega_{\Lambda}=0$ case in which, after COBE normalization of the power spectrum, there is a well known discrepancy between the amplitude required by $\sigma_{8}$ and the shape probed by redshift surveys: these two constraints favor different values of $n$. We find that the neutrino degeneracy can solve this problem with $\xi \gtrsim 3.5$; however, the allowed window is cut at $\xi \simeq 6$ by CMB data, and we are left with an interesting region in which $\Omega_{0}=1$ models are viable. This result is consistent with [9]. However, current evidences for a low $\Omega_{0}$ Universe [20,28] are independent of the constraints used here, so there are not many motivations at the moment to consider this window seriously.

\section{CONCLUSIONS}

We have considered some cosmological implications of a large relic neutrino degeneracy. We have shown that this degeneracy enhances the contribution of massive neutrinos to the present energy density of the Universe. For instance, neutrinos with a small mass $m_{\nu} \sim 10^{-2} \mathrm{eV}$ can contribute significantly to $\Omega_{0}$, provided that there is a large neutrino-antineutrino asymmetry.

Our main result is the computation of the power spectra of CMB anisotropies and matter density in presence of a neutrino degeneracy. We found, in agreement with [9], that it boosts the amplitude of the first CMB peak, shifts the peaks to larger multipoles, and supresses small scale matter fluctuations. These effects follow the increase of neutrino energy density, that delays matter-radiation equality.

We extended the calculation to the case of massive degenerate neutrinos, and showed the results for a mass of 0.07 $\mathrm{eV}$, as suggested by the Super-Kamiokande experiment. This mass has a small effect on CMB anisotropies. Indeed,

\footnotetext{
${ }^{5}$ The Viana \& Liddle result [22] is in very good agreement with an independent derivation by Girardi et al. 23].

${ }^{6}$ This confidence level stands for the goodness-of-fit of the model: when $\chi^{2}$ is greater than some value, the probability that we find the observed dataset, assuming the model to be valid, is smaller than $5 \%$. For the APM data, we have $9-3$ degrees of freedom, and the limiting value is found in numerical tables to be $\chi^{2}=12.5$.

${ }^{7}$ Here we have $19-3$ d.o.f.; the $95 \%$ CL is given by $\chi^{2}=26$.

${ }^{8}$ At least, for the values of the other cosmological parameters considered here. This situation can be easily improved, for instance, with $h=0.7$.
} 
such light neutrinos are still relativistic at recombination, but in presence of a degeneracy, they can account for a substantial part of the density today, of order $\Omega_{\nu} \sim 10^{-2}$. Also, we showed that small scale matter fluctuations are much more suppressed when the degenerate neutrinos are massive, because free-streaming of non-relativistic neutrinos is more efficient when their average momentum is boosted by the chemical potential.

We compared our results with observations, in the restricted case of a flat universe with arbitrary $\left(\Omega_{\Lambda}, \xi, n\right)$ and fixed values of other cosmological parameters. We found that for $\Omega_{\Lambda} \simeq 0.5-0.6$, a large degeneracy is allowed, up to $\xi \simeq 3$. However, this upper bound is smaller than the value $\xi \simeq 4.6$ needed to explain the generation of ultra-high energy cosmic rays by the annihilation of high-energetic neutrinos on relic neutrinos with mass $m_{\nu}=0.07 \mathrm{eV}[29$. We also tried smaller values of $\Omega_{\Lambda}$, even if they are not favored by combined CMB and supernovae data. It turns out that a large degeneracy can account for both CMB and LSS constraints even with $\Omega_{0}=1$, provided that $3.5 \leq \xi \leq 6$. This analysis could be extended to other cosmological models. For instance, the degeneracy is likely to be compatible with a large contribution of tensor perturbations to large scale CMB anisotropies.

Finally, it turns out that the degeneracy parameter and the mass of degenerate neutrinos have effects within the level of detectability of future CMB observations and redshift surveys, even with $m_{\nu} \sim 0.07 \mathrm{eV}$. However, a careful analysis should be performed in order to detect possible parameter degeneracy between $\xi, m_{\nu}$ and other cosmological parameters.

\section{ACKNOWLEDGEMENTS}

We thank S. Borgani, A. Masiero and A.Yu. Smirnov for useful discussions, as well as W. Kinney and A. Riotto for correspondence. This work has been supported by INFN and by the TMR network grant ERBFMRXCT960090.

[1] I. Affleck and M. Dine, Nucl. Phys. B249, 361 (1985)

[2] A. Casas, W.Y. Cheng and G. Gelmini, Nucl. Phys. B538, 297 (1999)

[3] R. Foot, M.J. Thomson and R.R. Volkas, Phys. Rev. D53, 5349 (1996)

[4] A.D. Linde, Phys. Rev. D14, 3345 (1976); J. Liu and G. Segre, Phys. Lett. B338, 259 (1994); B. Bajc, A. Riotto and G. Senjanović, Phys. Rev. Lett. 81, 1355 (1998)

[5] H.E. Haber and H.A. Weldon, Phys. Rev. D25, 502 (1982); A. Riotto and G. Senjanović, Phys. Rev. Lett. 79, 349 (1997)

[6] Y. Fukuda et al. [Super-Kamiokande Collaboration], Phys. Rev. Lett. 81, 1562 (1998)

[7] C.H. Lineweaver, Astrophys. J. 505, L69 (1998)

[8] G.B. Larsen and J. Madsen, Phys. Rev. D52, 4282 (1995)

[9] J.A. Adams and S. Sarkar, preprint OUTP-98-70P and talk presented at the workshop on The Physics of Relic Neutrinos, Trieste, September 1998

[10] W.H. Kinney and A. Riotto, preprint hep-ph/9903459

[11] U. Seljak and M. Zaldarriaga, Astrophys. J. 469, 437 (1996)

[12] K. Freese, E.W. Kolb and M.S. Turner, Phys. Rev. D27, 1689 (1983)

[13] H. Kang and G. Steigman, Nucl. Phys. B372, 494 (1992)

[14] P.B. Pal and K. Kar, Phys. Lett. B451, 136 (1999)

[15] S. Sarkar, Rept. Prog. Phys. 59, 1493 (1996) and references therein

[16] C. Ma and E. Bertschinger, Astrophys. J. 455, 7 (1995)

[17] W.T. Hu, Ph.D. Thesis (1995), preprint astro-ph/9508126

[18] D.J. Eisenstein, W. Hu and M. Tegmark, Astrophys. J. 518, 2 (1999)

[19] W. Hu, D.J. Eisenstein and M. Tegmark, Phys. Rev. Lett. 80, 5255 (1998)

[20] S. Perlmutter et al., Nature 391, 51 (1998); P.M. Garnavich et al., Astrophys. J. 493, L53 (1998); A.G. Riess et al., Astron. J. 116, 1009 (1998)

[21] L. Wang, R.R. Caldwell, J.P. Ostriker, and P.J. Steinhardt, preprint astro-ph/9901388

[22] P. Viana and A.R. Liddle, MNRAS 303, 535 (1999)

[23] M. Girardi, S. Borgani, G. Giuricin, F. Mardirossian and M. Mezzetti, Astrophys. J. 506, 45 (1998)

[24] J. Loveday, G. Efstathiou, B.A. Peterson, and S.J. Maddox, Astrophys. J. 400, L43 (1992)

[25] T. Kolatt and A. Dekel, Astrophys. J. 479, 592 (1997)

[26] J. Lesgourgues, D. Polarski and A.A. Starobinsky, MNRAS 308, 281 (1999)

[27] C.H. Lineweaver and D. Barbosa, Astron. \& Astroph. 329, 799 (1998); Astrophys. J. 496, 624 (1998) 
[28] N.A. Bahcall, X. Fan, and R. Cen, Astrophys. J. 485, L53 (1997); X. Fan, N.A. Bahcall, and R. Cen, Astrophys. J. 490, L123 (1997); V.R. Eke, S. Cole, C.S. Frenk, and J.P. Henry, MNRAS 298, 1145 (1998)

[29] G. Gelmini and A. Kusenko, Phys. Rev. Lett. 82, 5202 (1999) 
FIG. 1. Present energy density of massive degenerate neutrinos as a function of the degeneracy $\xi$. The curves correspond to different values of $h^{2} \Omega_{\nu}$ and the horizontal line is the upper bound from eq. (6).

FIG. 2. Same as figure 1 for the neutrino asymmetry $L_{\nu}$.

FIG. 3. CMB anisotropy spectrum for different models with one family of degenerate neutrinos. Solid lines account for the case of massless degenerate neutrinos, and correspond, from bottom to top, to $\xi=0,3,5$. Dashed lines correspond to degenerate neutrinos with mass $m_{\nu}=0.07 \mathrm{eV}$. Other parameters are fixed to $h=0.65, \Omega_{b}=0.05, \Omega_{\Lambda}=0.70, \Omega_{C D M}=1-\Omega_{b}-\Omega_{\nu}-\Omega_{\Lambda}$, $Q_{r m s-p s}=18 \mu \mathrm{K}, n=1$. We neglect reionization and tensor contribution.

FIG. 4. Present power spectrum of matter density, for the same parameters as in the previous figure. From top to bottom, to $\xi=0,3,5$.

FIG. 5. LSS and CMB constraints in $(\xi, n)$ space, for different choices of $\Omega_{\Lambda}$ : from top left to bottom right, $\Omega_{\Lambda}=0,0.5,0.6,0.7$. The underlying cosmological model is flat, with $h=0.65, \Omega_{b}=0.05, Q_{r m s-p s}=18 \mu \mathrm{K}$, no reionization, no tensor contribution. The allowed regions are those where the labels are. For LSS constraints, we can distinguish between degenerate neutrinos with $m_{\nu}=0$ (solid lines) and $m_{\nu}=0.07 \mathrm{eV}$ (dotted lines). 


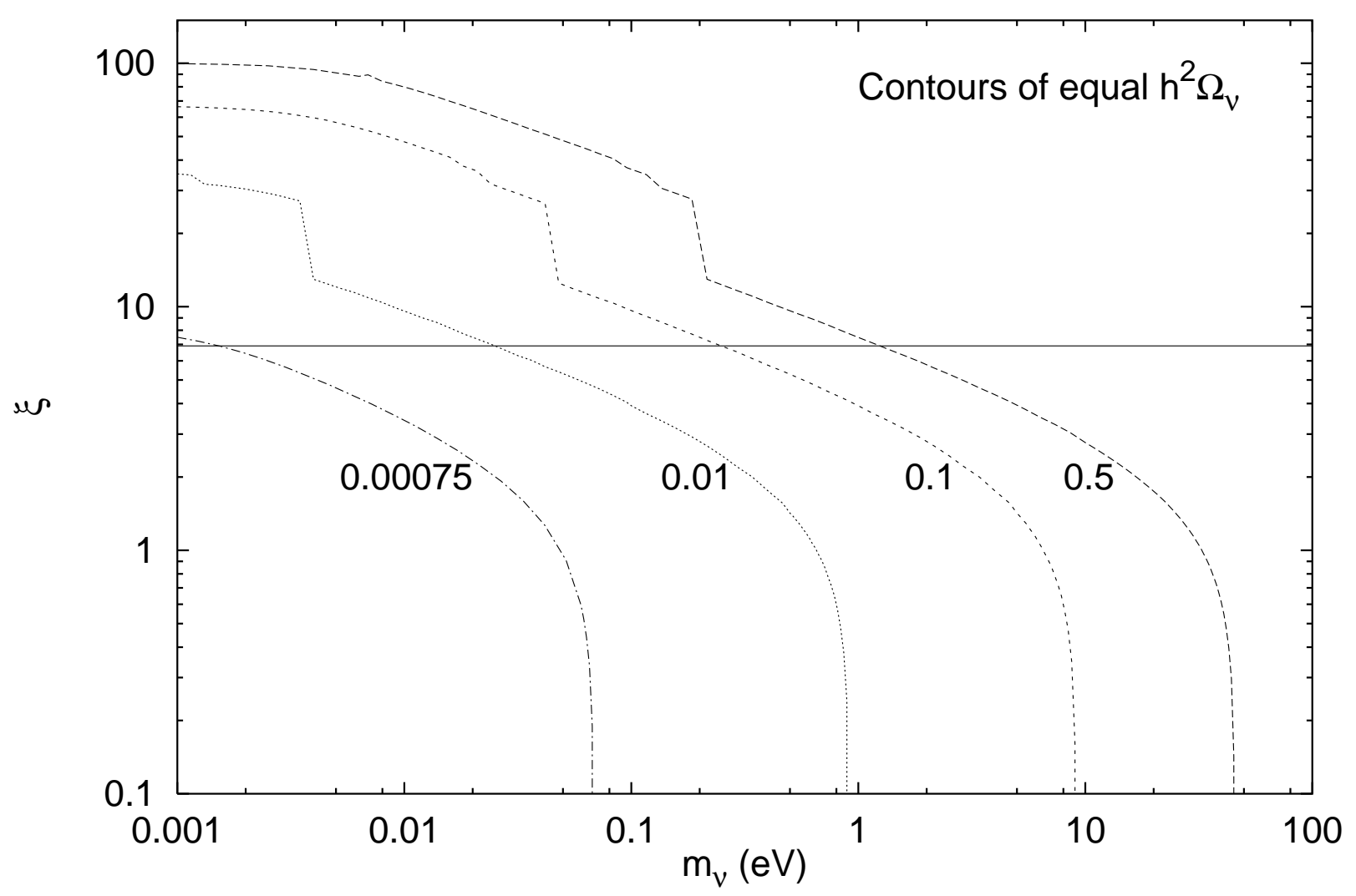

Figure 1 


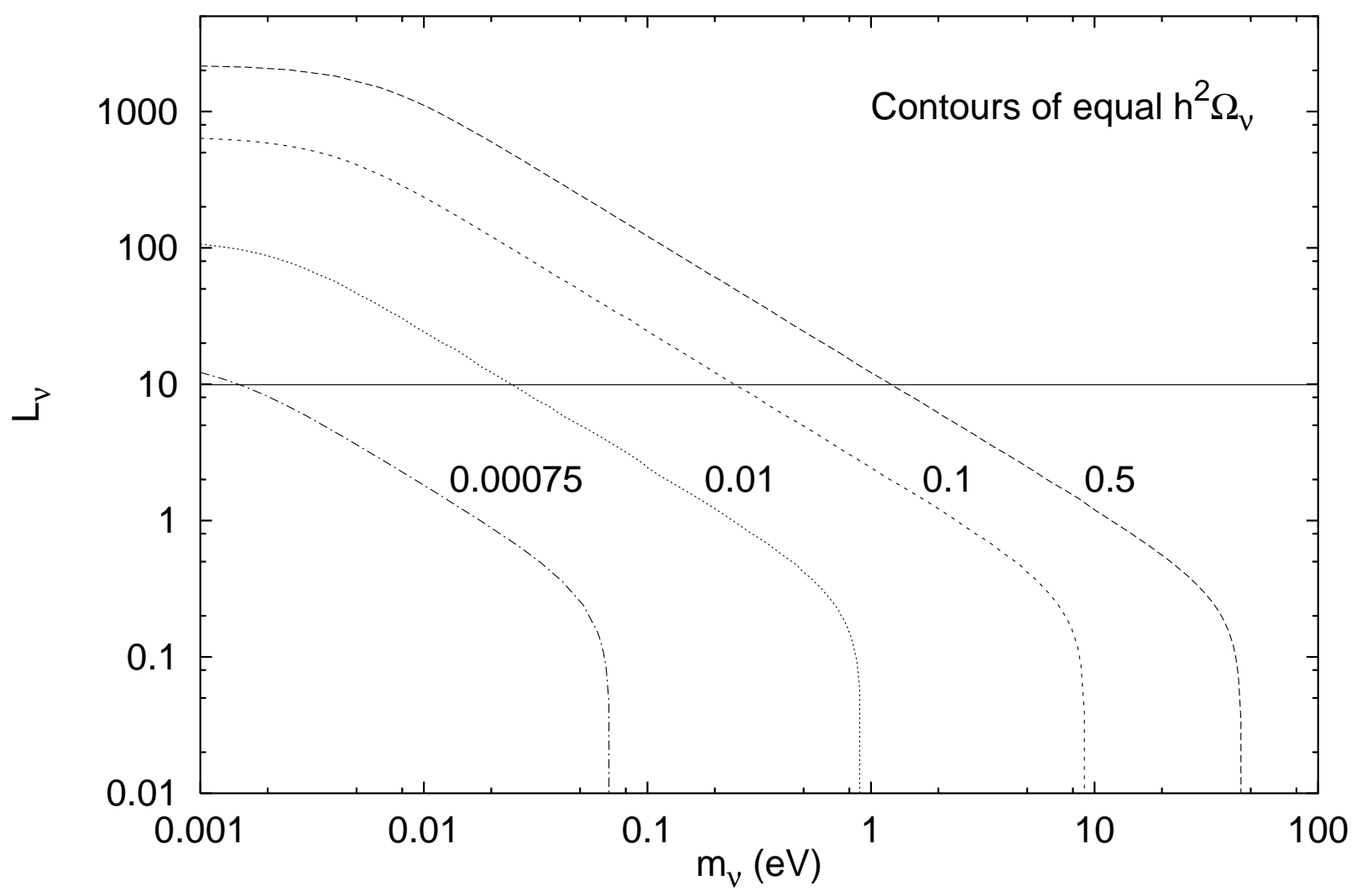

Figure 2 


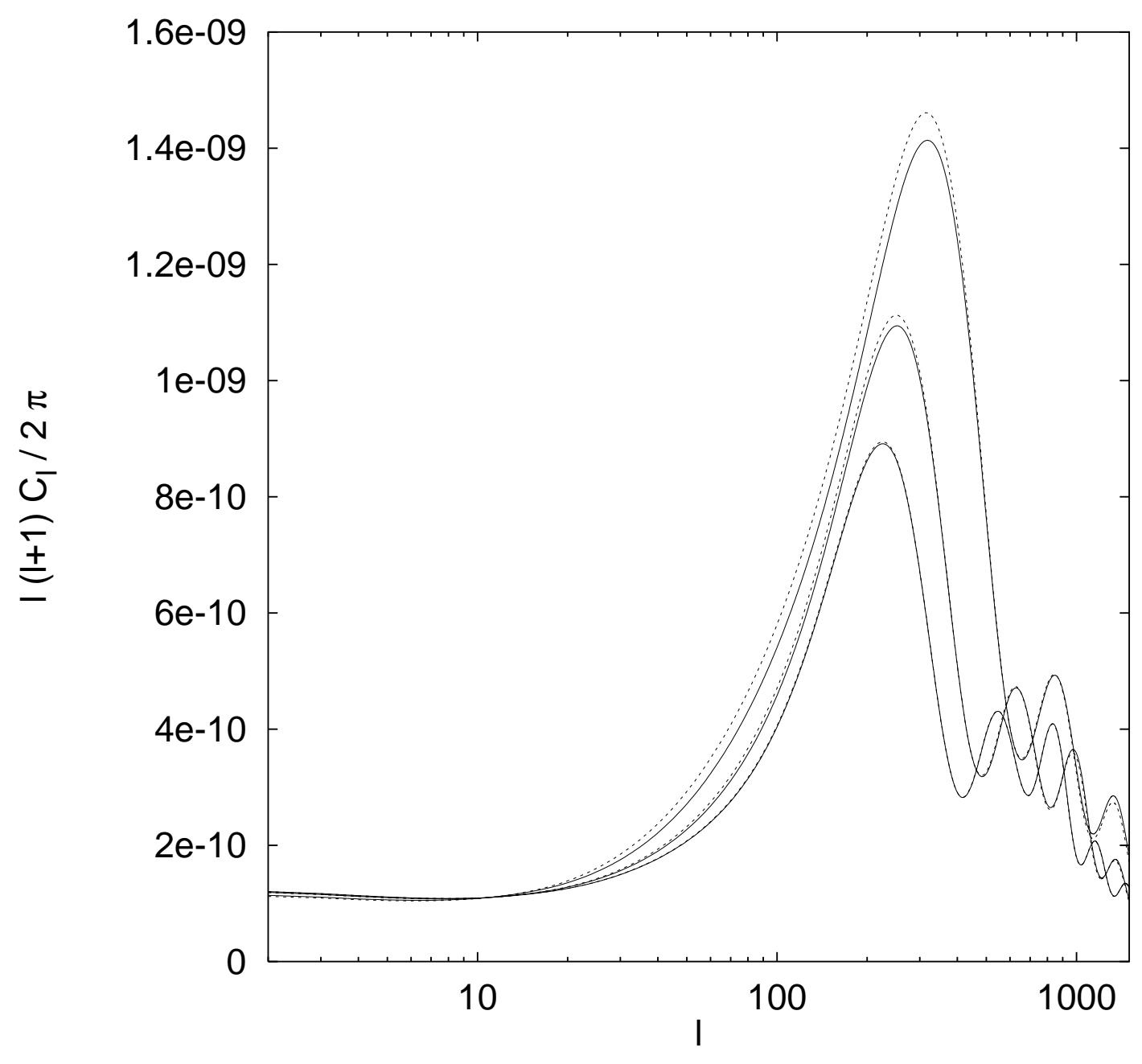

Figure 3 


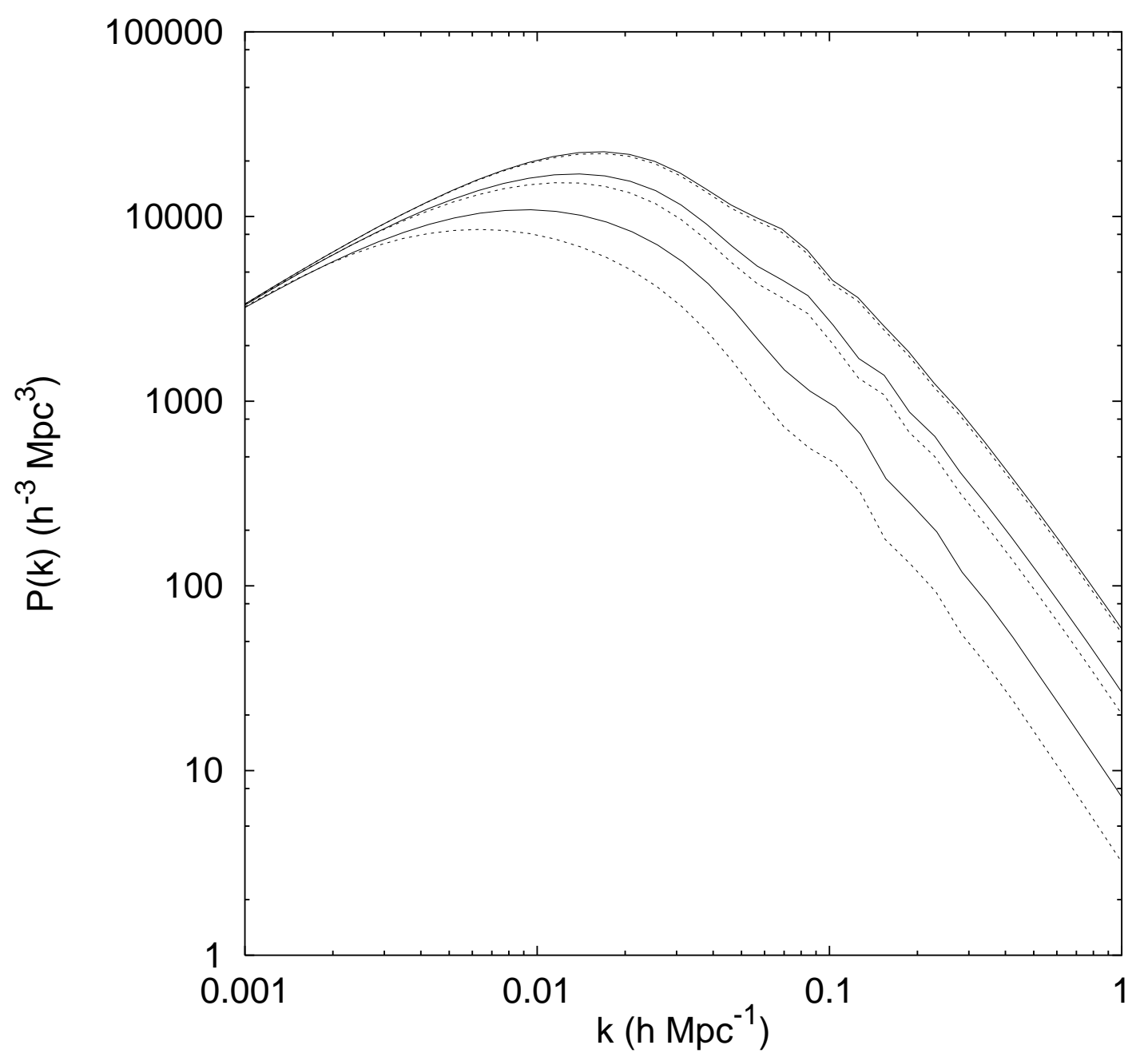

Figure 4 

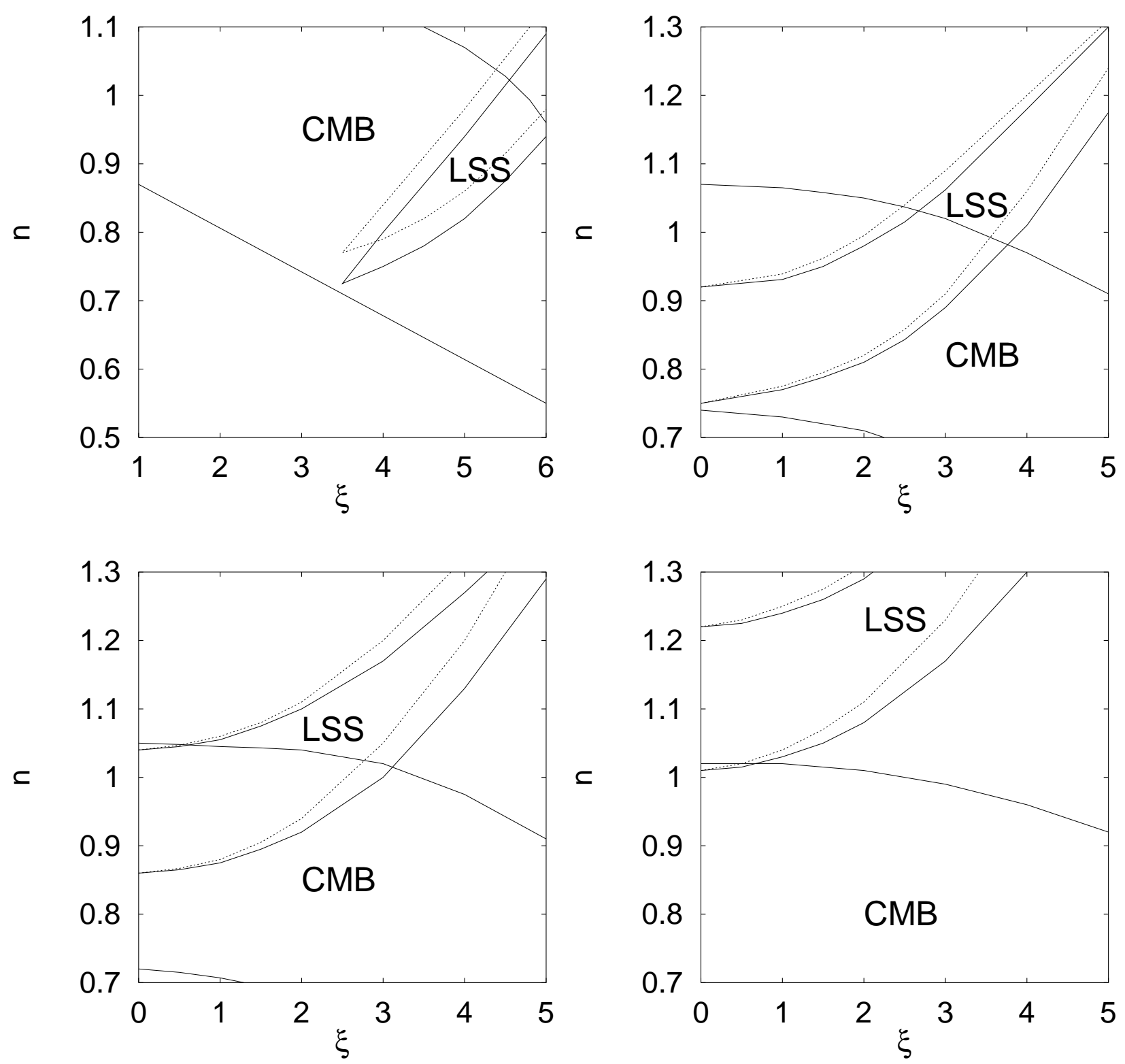

Figure 5 